\title{
Live-cell imaging and ultrastructural analysis reveal remarkable features of cultured porcine gonocytes
}

\begin{abstract}
Gonocytes in the neonatal testis have male germline stem cell potential. The objective of the present study was to examine the behavior and ultrastructure of gonocytes in culture. Neonatal porcine testis cells were cultured for 4 weeks and underwent live-cell imaging to explore realtime interactions among cultured cells. This included imaging every $1 \mathrm{~h}$ from day 0 to day 3 , every $2 \mathrm{~h}$ from day 4 to day 7, and every $1 \mathrm{~h}$ for $24 \mathrm{~h}$ at days 14, 21, and 28. Samples also underwent scanning electron microscopy, transmission electron microscopy, morphometric evaluations, immunofluorescence, and RT-PCR. Live-cell imaging revealed an active amoeboid-like movement of gonocytes, assisted by the formation of extensive cytoplasmic projections, which, using scanning electron microscopy, were categorized into spike-like filopodia, leaf-like lamellipodia, membrane ruffles, and cytoplasmic blebs. In the first week of culture, gonocytes formed loose attachments on top of a somatic cell monolayer and, in week 2, formed grape-like clusters, which, over time, grew in cell number. Starting at week 3 of culture, some of the gonocyte clusters transformed into large multinucleated embryoid bodylike colonies (EBLCs) that expressed both gonocyte- and pluripotent-specific markers. The number and diameter of individual gonocytes, the number and density of organelles within gonocytes, as well as the number and diameter of the EBLCs increased over time $(\mathrm{P}<0.05)$. In conclusion, cultured porcine gonocytes displayed extensive migratory behavior facilitated by their various cytoplasmic projections, propagated, and transformed into EBLCs that increased in size and complexity over time.
\end{abstract}

Keyword: Cell culture; Germline stem cells; Gonocytes; Live-cell imaging; Ultrastructure 